\title{
Relationship between semen characteristics and fertility in electroejaculated mice
}

\author{
R. A. Anderson, Jr*, C. Oswald*§, B. R. Willis $†$ and L. J. D. Zaneveld* $\ddagger$
}

Departments of ${ }^{*}$ Physiology and Biophysics, $\uparrow$ Anatomy and $\ddagger$ Obstetrics and Gynecology, University of Illinois at Chicago, Health Sciences Center, P.O. Box 6998, Chicago, Illinois 60680, U.S.A.

\begin{abstract}
Summary. Ejaculates were obtained from C57BL mice by applying two successive series of electrical stimuli which were delivered via a bipolar rectal probe. The ejaculates thus collected contained fertile spermatozoa as indicated by results from invitro fertilization. Once separated from the seminal plasma, ejaculated spermatozoa possessed the same in-vitro fertilization rate as epididymal sperm. Ejaculates were analysed for coagulum weight, ejaculate volume, sperm count, sperm motility, acid phosphatase content and fructose content. Significant differences were present between several of these values for fertile and infertile mice, and values were therefore empirically assigned to represent minimal amounts for 'normal' fertility $(1.5 \mu 1$ ejaculate volume; $10.2 \mathrm{mg}$ coagulum weight; $2.5 \times 10^{6}$ spermatozoa/ml; $2.3 \times 10^{3}$ motile spermatozoa/ejaculate). One half of the fertile animals had no deficiencies in any of the characteristics measured, whereas $97 \%$ of the infertile animals had at least one deficiency. No fertile male had more than 2 deficiencies. These data show that the characteristics of mouse semen obtained by the present method of electroejaculation are related to the fertility status of the animal.
\end{abstract}

\section{Introduction}

The mouse is a very useful laboratory animal for studies in reproductive physiology, especially those concerned with the effects of various drugs on fertility. Advantages for its use include: (1) its small size (and hence low cost); (2) its well-known reproductive cycle; and (3) its relatively short gestational period (Rugh, 1968; Monesi, 1978). However, there is a relatively high incidence of sterility in the mouse, especially in highly inbred strains, and meaningful data on the effects of a drug or treatment on fertility can only be obtained when animals of proven fertility are used. Reduced fertility should be a result of treatment, rather than due to chance. To date, the only effective measurement of fertility in the mouse has been through the use of mating experiments, which are time-consuming, as well as costly. Male mice could potentially be screened for fertility on the basis of semen analysis. Reports have appeared which describe the collection of mouse semen by electroejaculation (Scott \& Dziuk, 1959; Snyder, 1966). These methods are unsatisfactory, however, due to the poor quality of semen obtained, or to procedure-related mortality. The present report presents an improved method for the collection and analysis of mouse semen obtained by electroejaculation.

$\S$ Present address: Department of Biology, Indiana University, Bloomington, Indiana 47401, U.S.A.

$0022-4251 / 83 / 030001-07 \$ 02 \cdot 00 / 0$

(C) 1983 Journals of Reproduction \& Fertility Ltd 


\section{Materials and Methods}

\section{Materials}

The electrical stimulator (Model SAS-1; Nemco Nuclear, Bryan, Texas) consists of a sine wave rheostat transformer, possessing variable output of $0-15 \mathrm{~V}$ at $60 \mathrm{~Hz}$. A bipolar rectal electrode was constructed at the instrument facility of the University of Illinois at Chicago, Health Sciences Center. The probe was covered throughout its length with a polyethylene sheath, except for $3 \mathrm{~mm}$ sections at the tip and $7 \mathrm{~mm}$ from the tip, which were cut out to expose a stainless-steel and copper electrode, respectively. The diameter of the probe was $2 \mathrm{~mm}$.

Sorbitol dehydrogenase, nicotinamide adenine dinucleotide (reduced form) and p-nitrophenylphosphate were obtained from Sigma Chemical Company (St Louis, Missouri). Fructose was purchased from Fisher Scientific (Itasca, Illinois). All other reagents were of the highest quality commercially available.

\section{Mating tests}

Male C57BL/6J mice (60-70 days of age) were obtained from Jackson Laboratories (Bar Harbor, Maine). The normal frequency of infertility in these animals is $10-15 \%$ (records of Jackson Laboratories). To obtain a sufficient number of infertile animals for the present study, it was necessary to evaluate the fertility of a rather large number of males (225) through the use of in-vivo mating experiments. Based on these studies, a group of 53 animals was selected such that about two-thirds of the animals were infertile. This group of animals was used for further study. The animals were individually housed under constant lighting $(14 \mathrm{~h} \mathrm{light} / 10 \mathrm{~h}$ dark cycle; lights on at $05: 00 \mathrm{~h}$ and temperature $\left(23 \pm 0.5^{\circ} \mathrm{C}\right)$, and were given free access to laboratory chow and water. The fertility of each male was assessed as follows: 2 female mice (white Swiss) were placed with each male. The females were checked twice each day for the presence of a vaginal plug. The females were allowed to remain with the males for 5 days or until a vaginal plug was observed. If no vaginal plug was observed, the females were removed and the male allowed to rest for 2 days, after which 2 different females were placed in its cage. Each male was used as described, until 4 weeks had elapsed, or until a vaginal plug was detected in at least 3 females. Males were thus mated with as many as 8 different females over a period of up to 4 weeks for the evaluation of fertility. A male was considered fertile if progeny were sired.

\section{Electroejaculation}

The rectal probe was lubricated with $\mathrm{K}-\mathrm{Y}$ jelly, and inserted $2 \cdot 3 \mathrm{~cm}$ into the rectum of an unanaesthetized mouse. Stimuli of $2 \mathrm{sec}$ duration were applied in $1 \mathrm{~V}$ increments, starting at $1 \mathrm{~V}$, with a $2-s e c$ rest period between stimuli. Two stimuli were administered at each voltage setting, up to a maximum of $5 \mathrm{~V}$, or until a coagulum was detected at the tip of the glans penis, whichever occurred first. This procedure was repeated until either a maximum stimulus of $5 \mathrm{~V}$ was delivered, or until a second coagulum was produced. Administration of two sets of stimuli produced the best quality semen with the minimum amount of apparent discomfort to the animal. Throughout the stimulation period, semen appearing at the tip of the glans penis was collected with a $25 \mu$ l capillary pipette. The coagulum was removed from the glans penis with forceps. It was especially important to remove the coagulum completely so that blockage of the urethra, with subsequent uraemic poisoning, did not occur. The combined fluid and coagula resulting from the 2 sets of electrical stimuli were regarded as the ejaculate.

\section{Semen analysis}

The volume of the ejaculate was determined by measuring the fractional length of the capillary pipette which was filled by semen. Due to the small volume, the semen was subsequently diluted in 
9 volumes of Krebs-Ringer Hepes buffer $\left(0 \cdot 12 \mathrm{M}-\mathrm{NaCl}, 4.3 \mathrm{~mm}-\mathrm{KCl}, 1.2 \mathrm{~mm}-\mathrm{MgSO}_{4}, 2 \mathrm{~mm}\right.$ -

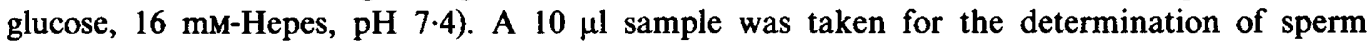
concentration and percentage sperm motility. The sperm concentration was determined with a Neubauer haemocytometer (Zaneveld \& Polakoski, 1977). The percentage of motile spermatozoa was evaluated by direct microscopic observation. The number of motile spermatozoa in the ejaculate was calculated from the sperm concentration, ejaculate volume and the estimated percentage sperm motility. Each coagulum was weighed to the nearest $0.1 \mathrm{mg}$. When 2 coagula were produced, the combined weights were taken as the coagulum weight.

The remainder of each diluted ejaculate was used to determine either the fructose or acid phosphatase content. Fructose was determined enzymically by comparing the initial rate of NADH oxidation in the presence of the sample and sorbitol dehydrogenase with the rates obtained with fructose standards (Anderson, Reddy, Oswald \& Zaneveld, 1979). Acid phosphatase was measured by a standard clinical procedure (Kachman, 1970). The rate of release of $p$-nitrophenylphosphate at $\mathrm{pH} 5.0$ was determined spectrophotometrically $(410 \mathrm{~nm})$ after adjusting the $\mathrm{pH}$ of the reaction mixture to $9 \cdot 0$.

\section{In-vitro fertilization}

This was carried out as described previously (Anderson et al., 1980b). Briefly, epididymal spermatozoa were obtained from proven breeder C57BL mice by making small cuts in each cauda epididymidis, placing the epididymides in capacitation medium (modified Krebs-Ringer bicarbonate, containing $20 \mathrm{mg}$ bovine serum albumin $/ \mathrm{ml}$; Anderson et al., 1980b), allowing the spermatozoa to escape from the epididymal tubule, and removing the tissue by filtration through cheesecloth. Spermatozoa were incubated in the capacitation medium for $1 \mathrm{~h}$ before adding an aliquant of the sperm suspension to oocytes, obtained from the ampullae of the oviducts of superovulated sexually mature female (white Swiss) mice. To obtain sufficient numbers of spermatozoa, the ejaculates of 2 mice were pooled before capacitation. All incubations contained $2 \times 10^{4}$ spermatozoa. Ejaculated spermatozoa were capacitated in the presence or absence of seminal plasma. To separate the spermatozoa from the seminal plasma, the semen was diluted in 9 volumes of Krebs-Ringer Hepes buffer and centrifuged at $800 \mathrm{~g}$ for $5 \mathrm{~min}$; the supernatant was removed and the sedimented spermatozoa resuspended in $1 \mathrm{ml}$ capacitation medium. Oocytes were examined for signs of fertilization (a swollen decondensing sperm nucleus, 2 pronuclei or the presence of 2-celled embryos) after incubation for $24 \mathrm{~h}$ at $37^{\circ} \mathrm{C}$.

\section{Statistical analyses}

Semen volume, coagulum weight, fructose concentration and acid phosphatase content were expressed as the mean \pm s.e.m. Due to the Poisson distribution of the sperm counts, the data for sperm concentration and motile spermatozoa per ejaculate were normalized by logarithmic transformation before further parametric analyses (Sokal \& Rohlf, 1969). Values were expressed as the antilogarithm of the average log value, with the $90 \%$ confidence limits indicated in parentheses. Data concerned with the percentage of motile spermatozoa were normalized by applying an arcsine transformation in which percentage was converted to the decimal equivalent, and the arcsine of the square root of this value was calculated. Data were expressed as the backtransform of the average arcsine transformation, with the $90 \%$ confidence limits indicated in parentheses. Differences among groups were evaluated by one-way analysis of variance of the measured data (volume, coagulum weight, fructose, acid phosphatase) or the transformed data (sperm count, motile spermatozoa per ejaculate, $\%$ motility). If one-way analysis of variance indicated a significant $(P<$ 0.05 ) difference among groups, the data were further analysed by the Newman-Keuls multiple range test (Woolf, 1968). Data concerned with the frequency of animals displaying one or more deficiencies in the semen characteristics were evaluated by $\chi^{2}$ analysis. In all cases, differences were considered significant at the 0.05 level. 


\section{Results}

\section{Electroejaculation}

Based upon the results from the mating studies, 52 mice were distinguished as (1) those that sired progeny ('fertile' animals); (2) those that did not sire progeny, but apparently had copulated, as indicated by the presence of a vaginal plug in the females with which they were mated ('infertile' animals); and (3) those that neither sired progeny nor provided evidence of copulation ('nonmating' animals). The last two groups were collectively termed 'non-breeding' animals.

The method used for electroejaculation yielded semen in a high percentage of the mice and was accompanied by very low mortality. Only one animal died after a total of 192 successful ejaculations of 53 mice, representing a mortality rate of approximately $0.5 \%$. The presence of a distended bladder in this animal suggested that the cause of death was uraemic poisoning. This may have the result of incomplete removal of the coagulum from the urethra after ejaculation. Only 7 attempts ( 6 of which were with infertile or non-mating mice) failed to produce an ejaculate, representing a $96.4 \%$ success rate. To obtain a semen sample with adequate numbers of motile spermatozoa, two sets of electrical stimuli were administered. During the first stimulation, some semen was ejaculated, but it did not contain any spermatozoa, or only very small numbers of immotile spermatozoa. During the second stimulation, motile spermatozoa were produced. Due to the potentially injurious nature of electrostimulation, no attempts were made to stimulate the animals more than twice.

\section{In-vitro fertilization}

The fertilizing capacity of ejaculated spermatozoa from mice of proven fertility was compared to that of spermatozoa obtained from the epididymis. The fertilization rate of epididymal spermatozoa was $60 \%$ (range $=48-80 \% ; n=8$ ). In contrast, no fertilization occurred when whole semen was used as a source of spermatozoa $(n=4)$. However, if the seminal plasma was first removed from the ejaculated spermatozoa by centrifugation, and the spermatozoa resuspended in medium and capacitated, the fertilization rate $(42 \%$; range $34-53 \% ; n=4)$ was essentially identical to that of epididymal spermatozoa that were centrifuged and resuspended in capacitation medium $(47 \%$; range $36-56 \% ; n=4)$. A decrease in sperm motility after centrifugation was the probable cause of the lower fertilization rates compared to those for uncentrifuged epididymal spermatozoa.

\section{Semen characteristics and the fertility status of mice}

The semen analyses for the three groups of mice are summarized in Table 1. The sperm concentration and the total number of motile spermatozoa per ejaculate were significantly lower in the infertile and non-mating mice than in the fertile mice. The average coagulum weight of the nonmating mice was significantly lower than that of the fertile mice. There were no statistically significant differences in the fructose or acid phosphatase content of semen obtained from any of the three groups, although the non-mating mice tended to have lower fructose levels than did the fertile mice.

On the basis of the semen analyses and fertility of individual animals, lower limits for ejaculate volume, coagulum weight, sperm count and number of motile spermatozoa per ejaculate were empirically determined as $1.5 \mu \mathrm{l}, 10.2 \mathrm{mg}, 2.5 \times 10^{6} / \mathrm{ml}$ and $2.3 \times 10^{3} /$ ejaculate, respectively. No correlation was observed between fertility and fructose or acid phosphatase content in these groups of animals. A value was considered deficient if it fell below the lower limits which were established.

Deficiencies were observed in a higher proportion of infertile and non-mating (non-breeders) mice than of fertile mice. A significantly higher proportion $\left(P<0.005 ; \chi^{2}\right.$ analysis $)$ of non-breeders showed deficiencies in coagulum weight, sperm count, and total motile spermatozoa per ejaculate (Table 2). As a function of the number of deficiencies per ejaculate, the distribution of breeders was substantially different from that of non-breeders (Table 3). The greatest frequency of non-breeders had three semen deficiencies, whereas semen samples with zero deficiencies was the mode for the animals with proven fertility. 
Table 1. Characteristics of electroejaculated semen

\begin{tabular}{|c|c|c|c|}
\hline Characteristic & Fertile & Infertile & Non-mating \\
\hline $\begin{array}{l}\text { No. of mice } \\
\text { Ejaculate }\end{array}$ & 18 & 13 & 21 \\
\hline volume $(\mu \mathrm{l})$ & $3 \cdot 5 \pm 0 \cdot 3$ & $3.0 \pm 0.2$ & $3.6 \pm 0.3$ \\
\hline $\begin{array}{l}\text { Coagulum wt (mg) } \\
\text { Sperm conc.* }\end{array}$ & $14.0 \pm 1.8$ & $11.4 \pm 1.2$ & $7 \cdot 4 \pm 1.3 \ddagger$ \\
\hline $\begin{array}{l}\text { Sperm conc. } \\
\left(\times 10^{-6} / \mathrm{ml}^{-}\right) \\
\text {Motile spermatozoa }\end{array}$ & $4 \cdot 5(1.4-14 \cdot 0)$ & $1.8(0.4-7.6)+$ & $1.4(0.2-10 \cdot 0) \ddagger$ \\
\hline $\begin{array}{l}\left(\times 10^{-3} / \text { ejaculate }\right) \\
\% \text { motility } \\
\text { Acid phosphatase }\end{array}$ & $\begin{array}{l}3 \cdot 5(0 \cdot 7-17 \cdot 7) \\
42(8-82)\end{array}$ & $\begin{array}{l}1 \cdot 3(0 \cdot 2-10 \cdot 6) \ddagger \\
29(3-66)\end{array}$ & $\begin{array}{l}0 \cdot 8(0 \cdot 2-3 \cdot 0) \ddagger \\
28(2-70)\end{array}$ \\
\hline $\begin{array}{l}(\mathrm{mi} . \mathrm{u} . / \mathrm{ml}) \\
\text { Fructose (mM) }\end{array}$ & $\begin{array}{l}69 \pm 8 \\
21 \pm 5\end{array}$ & $\begin{array}{l}67 \pm 11 \\
30 \pm 9\end{array}$ & $\begin{array}{l}68 \pm 6 \\
10 \pm 2\end{array}$ \\
\hline
\end{tabular}

Values are the mean \pm s.e.m. or mean with $90 \%$ confidence limits in parentheses.

* Data were transformed to log (values) before parametric statistical analysis (Sokal \& Rohlf, 1969).

$\dagger$ Data were subjected to arcsine transformation before parametric statistical analysis (Sokal \& Rohlf, 1969).

$\ddagger$ Value differs significantly from that of fertile mice $(P<0.05)$, Newman-Keuls multiple range test).

Table 2. Frequencies of deficiencies in individual semen characteristics

\begin{tabular}{lccccc}
\hline & & \multicolumn{4}{c}{ Deficiency frequency $(\%)$} \\
\cline { 3 - 6 } & $\begin{array}{c}\text { No. of } \\
\text { mice }\end{array}$ & $\begin{array}{c}\text { Ejaculate } \\
\text { vol. }\end{array}$ & $\begin{array}{c}\text { Coagulum } \\
\text { wt }\end{array}$ & $\begin{array}{c}\text { Sperm } \\
\text { conc. }\end{array}$ & $\begin{array}{c}\text { Total no. } \\
\text { of motile spermatozoa }\end{array}$ \\
\hline Fertile & 18 & 0 & 28 & 22 & 28 \\
Infertile & 13 & 8 & $62^{*}$ & $69^{*}$ & $69^{*}$ \\
Non-mating & 21 & 10 & $76^{*}$ & $71^{*}$ & $71^{*}$ \\
\hline
\end{tabular}

Semen analyses from individual animals were compared with empirically determined minimal values for 'normal' fertility (see text). Any value falling below the established minimal values was considered a deficiency.

* Differs from value for fertile animals $\left(P<0.005 ; \chi^{2}\right.$ analysis $)$.

Table 3. Cumulative distribution of semen deficiencies in fertile and non-breeding mice

\begin{tabular}{ccc}
\hline & \multicolumn{2}{c}{$\begin{array}{c}\text { Mice with deficient semen characteristics } \\
\text { (cumulative \%) }\end{array}$} \\
\cline { 2 - 3 } No. of deficiencies & Fertile $(n=18)$ & Non-breeding $(n=34)$ \\
\hline 0 & $9(50)$ & $1(3)^{*}$ \\
1 & $6(83)$ & $8(26)^{*}$ \\
2 & $3(100)$ & $8(50)^{*}$ \\
3 & $0(100)$ & $16(97)$ \\
4 & $0(100)$ & $13(100)$ \\
\hline
\end{tabular}

* Differs from value for fertile mice $\left(P<0.001 ; \chi^{2}\right.$ analysis).

From these data, fertile mice could be selected on the basis of the number of deficient variables measured in semen collected by electroejaculation. In the present study, $97 \%$ of the non-breeders could be eliminated by using only those animals which produced semen with no deficient variables. On the other hand, if animals with up to 2 deficiencies were used, $50 \%$ of the non-breeder animals would be rejected, while retaining $100 \%$ of the fertile animals of the initial population (Table 3 ). 


\section{Discussion}

The present method of electroejaculation was very successful and associated with a low mortality rate. Although the sperm numbers are relatively low, the spermatozoa are of reasonable quality as evidenced by the sperm motility $(40-50 \%)$, which is essentially the same as that of epididymal spermatozoa $(47 \%$; range $=34-59 \% ; n=12)$. The mouse normally mates frequently with the female over a relatively short period, showing a number of intromissions. One of the reasons that frequent coitus is necessary may be that only a small number of spermatozoa are ejaculated each time. Snyder (1966) reported that there was a lower number of spermatozoa in mouse semen obtained by electroejaculation when a coagulum was produced (i.e. under physiological conditions).

Electroejaculated spermatozoa obtained from fertile males by this method are capable of fertilizing mouse oocytes in vitro. Data from in-vitro fertilization experiments with electroejaculated spermatozoa suggest that mouse seminal plasma contains substances that may prevent the capacitation of spermatozoa. Apparently, antifertility substances are present in mouse seminal plasma, as they are in other species (McRorie \& Williams, 1974). Additionally, the quality of the semen varied with the fertility status of the male (Table 3 ). These data suggest that the electroejaculation technique produces semen samples that reflect the quality of the ejaculates produced during coitus.

Two methods of electroejaculation of the mouse had been described before the present study. These methods, however, had problems that the present study has attempted to minimize. Synder (1966) described a method whereby an entirely liquid ejaculate (no coagulum) was produced. Although the sperm count was higher than that reported in the present study, no measure of sperm viability was reported (e.g. motility, fertilizing capacity). Moreover, the extreme electrical stimuli which were used $(50-100 \mathrm{~V})$ resulted in $6 \%$ immediate mortality from electrocution, $16 \%$ additional deaths over the next 2 weeks, and $12 \%$ unsuccessful ejaculations. The mortality observed in the present study was much lower. Scott \& Dziuk (1959) employed lower electrical stimuli (10-15 V), but the ejaculates contained spermatozoa which were partly or completely entrapped within the coagulum. Additionally, the spermatozoa tended to be ejaculated in highly concentrated clumps, were poorly motile, and incapable of fertilizing in vivo. No further analysis of semen collected by this method was reported.

To our knowledge, no estimate of seminal fructose or acid phosphatase has been previously reported for the mouse. No correlation between fertility and fructose levels was established in the present study. However, evaluation of fructose (a secretory product of the seminal vesicles) could be of benefit in the evaluation of various treatments on fertility, since the seminal vesicles are an absolute requirement for fertility and fecundity in mice (Pang, Chow \& Wong, 1979).

Several semen characteristics differed significantly between males of proven fertility and nonbreeder animals (Table 1); the coagulum weight and fructose content were lower in semen from non-breeder males which showed no signs of copulation (vaginal plug) than in non-breeders which produced vaginal plugs. Diminished secretions from the coagulating gland (which includes fructose in the mouse; Mann, 1964) could at least partly explain the inability of some males to sire offspring.

Fertility was related to ejaculate volume, coagulum weight, sperm count and the number of motile spermatozoa per ejaculate (Table 2). These findings are based upon the distribution of animals whose semen analyses fell below empirically determined limits. The distribution of deficiencies in semen from males of proven fertility and from non-breeders permitted the selection of a group of animals whose incidence of infertility was greatly reduced. Analysis of electroejaculated semen from the C57BL mouse could provide a rapid empirical method for the elimination of virtually all $(97 \%$, in the present study) non-breeders from a large group of animals.

The method can be used to ascertain the fertility of mice before and after treatments which may affect reproductive function. If only those mice with no semen deficiencies were considered fertile, then non-breeder animals would probably be noticeable. However, the use of this criterion for 
fertility would inaccurately identify a rather large percentage of fertile males as being non-breeders. If, on the basis of semen analysis, a treatment was thought to have an adverse effect on fertility, other criteria (e.g. mating, in-vitro fertilization experiments) should be used to confirm the effect. On the other hand, there would be little likelihood of overlooking a potential contraceptive or chemosterilant on the basis of results obtained by the present method. Analysis of semen from the C57BL mouse could therefore be used to screen drugs and treatments for potentially adverse effects on male fertility. Although other strains of mice may be suitable for this purpose, normal (empirical) values of semen characteristics would have to be established. The present system has been used successfully to evaluate the adverse effects of chronic ethanol ingestion upon male reproductive function (Anderson et al., 1980a).

Supported by NIH grants AA 03521, AA 07374 and HD 09868. We thank Shari Roberts for assistance in the manuscript preparation.

\section{References}

Anderson, R.A., Reddy, J.M., Oswald, C. \& Zaneveld, L.J.D. (1979) Enzymic determination of fructose in seminal plasma by initial rate analysis. Clin. Chem. 25, 1780-1782.

Anderson, R.A., Reddy, J.M., Oswald, C. \& Zaneveld, L.J.D. (1980a) Decreased male fertility induced by chronic alcohol ingestion. Fedn Proc. Fedn Am. Socs exp. Biol. 39, 542.

Anderson, R.A., Willis, B.R., Oswald, C., Reddy, J.M., Beyler, S.A. \& Zaneveld, L.J.D. (1980b) Hormonal imbalance and alterations in testicular morphology induced by chronic ingestion of ethanol. Biochem. Pharmac. 29, 1409-1419.

Kachman, J.F. (1970) Enzymes. In Fundamentals of Clinical Chemistry, pp. 362-473. Ed. N. W. Tietz. W. B. Saunders, Philadelphia.

Mann, T. (1964) The Biochemistry of Human Semen and of the Male Reproductive Tract, pp. 37-52; 241. Methuen and Co., London.

McRorie, R.A. \& Williams, W.L. (1974) Biochemistry of mammalian fertilization. Ann. Review Biochem. 43, 777-803.

Monesi, V. (1978) Spermatogenesis and spermatozoa. In Reproduction in Mammals. I. Germ Cells and Fertiliza- tion, pp. 46-84. Eds C. R. Austin \& R. V. Short. Cambridge University Press.

Pang, S.F., Chow, P.H. \& Wong, T.M. (1979) The role of the seminal vesicles, coagulating glands and prostate glands on the fertility and fecundity of mice. $J$. Reprod. Fert. 56, 129-132.

Rugh, R. (1968) The Mouse, pp. 7-23. Burgess Publ. Co., Minneapolis.

Scott, J.V. \& Driuk, P.J. (1959) Evaluation of the electroejaculation technique and the spermatozoa thus obtained from rats, mice and guinea pigs. Anat. Rec. 133, 655-664.

Synder, R.L. (1966) Collection of mouse semen by electroejaculation. Anat. Rec. 155, 11-14.

Sokal, R.R. \& Rohlf, F.J. (1969) Biometry, pp. 386-387. W. H. Freeman, San Francisco.

Woolf, C.M. (1968) Principles of Biometry, pp. 101-109. D. Van Nostrand, Princeton.

Zaneveld, L.J.D. \& Polakoski, K.L. (1977) Collection and physical examination of the human ejaculate. In Techniques of Human Andrology, pp. 147-172. Ed. E. S. E. Hafez. Elsevier/North-Holland Biomedical Press, Amsterdam.

Received 22 June 1982 